\title{
Optical Coherence-Gated Imaging of Biological Tissues
}

\author{
Y. Pan, E. Lankenau, J. Welzel, R. Birngruber, and R. Engelhardt
}

\begin{abstract}
We present optical coherence-gated tomography (OCT) in turbid biological tissues. The fast OCT system that is used in this study is a single-mode fiber-optic interferometer with low-coherence light at $830 \mathrm{~nm}$ which can perform a crosssectional image of 250-600 pixels in a few seconds. Preliminary results suggest that $\mathrm{OCT}$ can provide high-resolution imaging in low-scattering and high-scattering superficial tissues. In vitro imaging of porcine cornea after being coagulated with a laser shows that OCT is a promising tool for the evaluation of pathological structures in low-scattering tissue. This technique can also be used to diagnose diseases in high-scattering tissues like bladder and living skin. In addition, $50-\mathrm{MHz}$ ultrasound images and histological pictures are presented for comparison with OCT.
\end{abstract}

\section{INTRODUCTION}

$\mathbf{N}$ ONINVASIVE OPTICAL imaging of living tissues, as an attractive alternative to the currently developed imaging modalities such as X-ray tomography, magneto-resonant tomography (MRT), and ultrasound imaging, has shown growing interest in early-stage diagnostics of tissue malformations such as tumors [1]. Optical methods have many advantages over conventional procedures in both safety and low costs. However, most biological tissues are characterized by the overwhelming light scattering in the "therapeutic window" of the wavelength range $(600 \sim 1300 \mathrm{~nm})$ which causes severely degraded imaging contrast and spatial resolution [2]. Conventional imaging techniques are therefore not applicable in most cases. In recent years, several new imaging reconstruction methods have been proposed to "see" through biological tissue. Time-resolved transillumination imaging has been used to localize breast tumors by detecting early-arriving photons and effectively rejecting multiple scattered light [3]-[5]. Phasemodulated techniques have been used for brain tomography to show oxygen metabolism [6]. Techniques such as CW acoustic modulated imaging [7] and phase-modulated and frequency-domain fluorescence imaging [8], [9] are currently being developed which may provide more specific imaging quality and diagnostic information.

This

work was supported in part by the Bundesministerium für Bildung, Wissenschaft, Forschung und Technologie (BMBF, FK-Nr.: 13N6302).

Y. Pan was with Medizinisches Laserzentrum Lübeck, D-23562 Lübeck, Germany. He is now with the National Science Foundation Center for Light Microscope, Imaging \& Biotechnology, Carnegie Mellon University, Pittsburgh, PA 15213 USA.

E. Lankenau, R. Birngruber, and R. Engelhardt are with Medizinisches Laserzentrum Lübeck, D-23562 Lübeck, Germany.

J. Welzel is with the Department of Dermatology, Medizinisches Laserzentrum Lübeck, D-23562 Lübeck, Germany.
Apart from the above photon migration imaging which can penetrate deep into the turbid tissue (a penetration of more than $5 \mathrm{~cm}$ with resolution of a few millimeters), there is a growing need and interest to show sub-50 $\mu \mathrm{m}$ microstructures in high-scattering superficial tissues such as human skin and some other structures like the bladder, cardiovascular system, and bronchus which are accessible endoscopically. Imaging of living skin by confocal microscopy which can visualize two-dimensional (2-D) and even three-dimensional (3-D) subcellular structures up to $200 \mu \mathrm{m}$ in depth has been recently reported [10]. However, because of the high scattering coefficient of skin, the exceptional capability of confocal microscopy for on-axis and lateral rejection of scattering from out of the micrometer-scale focal volume is limited to a maximal penetration depth of less than $300 \mu \mathrm{m}$. In many urological and dermatological applications however, high-resolution imaging into deeper layers is required to provide important diagnostic information. A new technique, i.e., coherence-gated imaging, including optical coherencegated tomography (OCT) [11], [12] and optical coherencegated microscopy (OCM) [13], can effectively reject multiple scattered light and extend the penetration depth down to 1 $\sim 2 \mathrm{~mm}$. In this paper, we will present cross-sectional OCT images of cornea, bladder, and living skin in comparison with high-frequency $(50 \mathrm{MHz})$ ultrasound and light microscopy and show the promising capabilities of this new technique for in vivo clinical diagnostics and presurgical aid.

\section{Methods AND THEORY}

The optical coherence-gated imaging system is an extension of optical coherence-domain reflectrometry (OCDR) [14] by performing additional lateral scans. By use of broad-band light sources such as superluminescence diodes or femtosecond lasers, this technique allows a noninvasive localization of 3-D reflecting sites with an axial and lateral resolution of $10-20 \mu \mathrm{m}$. An extremely high dynamic range in excess of $100 \mathrm{~dB}$ can be achieved by using optical heterodyne detection, which is capable of detecting ultraweak light signals in the order of femtowatts [15].

\section{A. Theoretical Considerations}

Analysis of coherent effects in a random medium has been a challenging problem in the field of optics for a long time. In a previous paper [16], we presented a theoretical model which supposed all the light fields reflected from the scattering 
medium and the reference mirror to give

$$
\begin{aligned}
I_{d}\left(L_{s}, L_{r}\right)= & I_{s}+I_{r}+2 \sqrt{I_{r} I_{s}} \int_{-\infty}^{\infty} \sqrt{R\left(L_{s}\right)}\left|V_{t c}(\Delta L)\right| \\
& \cdot \cos (\bar{k} \Delta L) \cdot d L_{s}
\end{aligned}
$$

where the interference modulation can be rewritten as

$$
I_{d}\left(L_{s}, L_{r}\right)=2 \sqrt{\left(I_{s} I_{r}\right)}\left[\sqrt{\left[R\left(L_{s}\right)\right]} \otimes C\left(L_{s}, L_{r}\right)\right] .
$$

$\Delta L=\left|L_{s}-L_{r}\right|, L_{s}=$ optical sample pathlength, $L_{r}=$ optical reference pathlength, $I_{s}=$ detected intensity backscattered by the sample, and $I_{r}=$ detected intensity from the reference arm. The normalized pathlength-resolved reflectance $R\left(L_{s}\right)$ is defined as $R\left(L_{s}\right)=\left[d I_{s}\left(L_{s}\right) / d L_{s}\right] / I_{s}$. $\otimes$ denotes the convolution operator $C\left(L_{s}, L_{r}\right)$, defined here as lowcoherence function, is equal to $\exp \left[-4\left(\left(L / L_{c}\right)^{2}\right] \cos k L_{s}\right.$ with the coherence length $L_{c}$ assuming that the spectral distribution of the light source has a Gaussian shape. Analysis of (2) leads to the conclusion that coherence-gated imaging traces out the variation of pathlength-resolved reflectance from microstructures with a difference in refractive index and shows speckle appearance because of the randomization of the reflected light field by scattering [16], [17]. In a further study [18], we measured the contrast of a razor blade inside Intralipid solutions with various scattering coefficients. There we figured out that the contrast strongly decreases at a depth of about six mean free paths (mfps) for that experimental setup. In other words, high light power may increase image acquisition speed but cannot further enhance the imaging contrast if it reaches the contrast limit caused by multiple scattering. Accordingly, the exceptional resolution of OCT will also be reduced with the increase of multiple scattering reentrance.

\section{B. Coherence-Gated Imaging Method}

Fig. 1 illustrates the setup of the single-mode fiberoptic coherence-gated imaging system. Light of a pigtailed, high-power broad-band superluminescence diode (SLD) is coupled into a single-mode fiberoptic interferometer. The central wavelength $\lambda$ of the SLD is $830 \mathrm{~nm}$ and the measured coherence length $L_{c}$ is $14 \mu \mathrm{m}$ (FWHM) in free space. Within the reference arm, the light is collimated by a gradiated index (GRIN) lens to a beam diameter of $0.7 \mathrm{~mm}$ and directed through a scanning mirror system to a reference mirror mounted on a stepper motor. The scanning mirror system is moved by a piezo electrical translator (PZT) and enables a scanlength of $2-3 \mathrm{~mm}$. The reference mirror determines the scan position. The PZT is driven with a constant speed in the axial direction which produces a Doppler frequency shift of the reflected light from the reference mirror. Optical heterodyne detection is used to obtain scan speeds of $10 \mathrm{~Hz} / \mathrm{A}$ and a high dynamic range of about $100 \mathrm{~dB}$ ((mirror signal)/stddev(noise)) of the interferometric signals.

The light in the sample arm is focused onto the spot of interest at the tissue sample. A computer controlled 2D translation stage is used to perform lateral scanning. By selecting $L_{S 1}$ and $L_{S 2}$ the system can be configured ei-

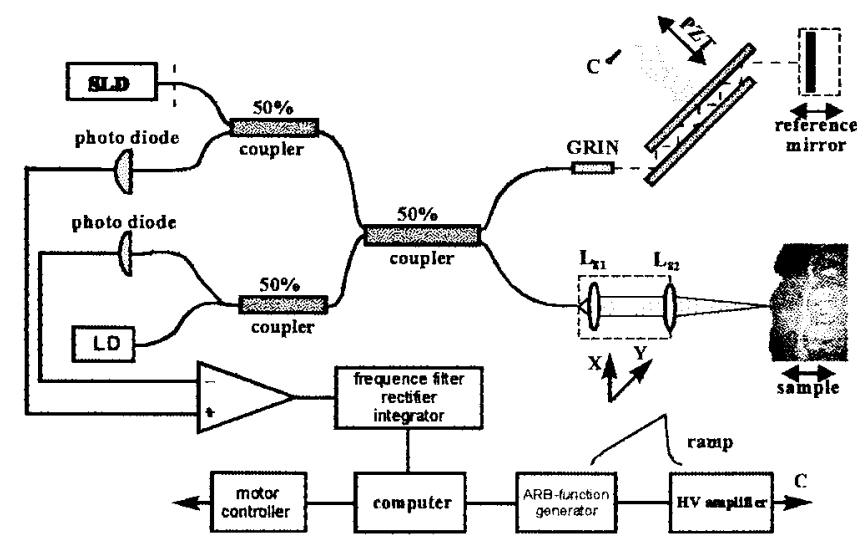

Fig. 1. Schematic diagram of a single-mode fiber-optic coherence-gated imaging system. SLD: pigtailed superluminescence diode $\left(\lambda_{0}=830 \mathrm{~nm}, \Delta \lambda=28 \mathrm{~nm}, P 0=2 \mathrm{~mW}\right)$. LD: red laser diode for alignment; GRIN: graded index lens; PZT: Piezoelectric translator $(\Delta d=180 \mu \mathrm{m})$.

ther as OCT (long-focus, narrow-beam incidence) or as an OCM (sharp-focus, broad-beam incidence) which combines coherence-gating and confocal features. The backscattered light from the tissue sample is collected through the same optics and recombined with reflected light from the scanning reference mirror system at two detectors. Coherent interference signal occurs only when the pathlength between these two arms are matched to within the coherence length $L_{c}$ of the light source. The dual-detector system and differential amplifier are used to minimize noise which is induced by the light source. After bandpass filtering the preamplified signal at the beat frequency (Doppler frequency), the amplitude of the interference signal is demodulated. The demodulated interference signal is transferred into the computer via a fast, high-resolution $\mathrm{A} / \mathrm{D}$ converter.

The axial resolution is determined by the coherence length of $L_{c} \approx 10 \mu \mathrm{m}$ in biological tissue whereas the lateral resolution is given by the focal spot size which is about $15 \mu \mathrm{m}$. Currently an image of $250-600$ pixels is performed in a few seconds. The system is able to acquire 2-D or 3-D data sets which can be displayed as false color or gray-scale images. The cross-sectional images of tissue microstructures are constructed from multiple scans of backscattered light intensity versus depth. The system has a SNR of $100 \mathrm{~dB}$ using a power of $230 \mu \mathrm{W}$ in the sample arm.

\section{RESUlTS AND DisCUSSION}

According to the above analyses, coherence-gated techniques have the exceptional capabilities of noninvasive, highresolution imaging in either low-scattering tissue like ocular structures or high-scattering superficial tissues like skin and endoscopically accessible organs such as the bladder, the bronchus, and vascular tissue. OCT has been extensively utilized for in vivo imaging of the retina and the anterior eye, however, only a few works are reported for in vivo imaging of highly scattering human tissue so far [12], [17], [19]-[22]. Below, we will first present OCT images in comparison with ultrasound and light microscopy, then indicate some results for the diagnostics of skin diseases. 


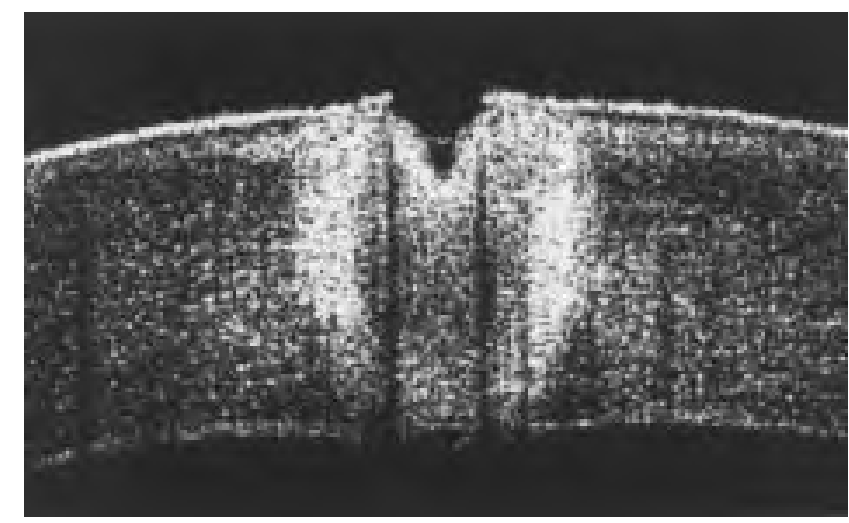

(a)

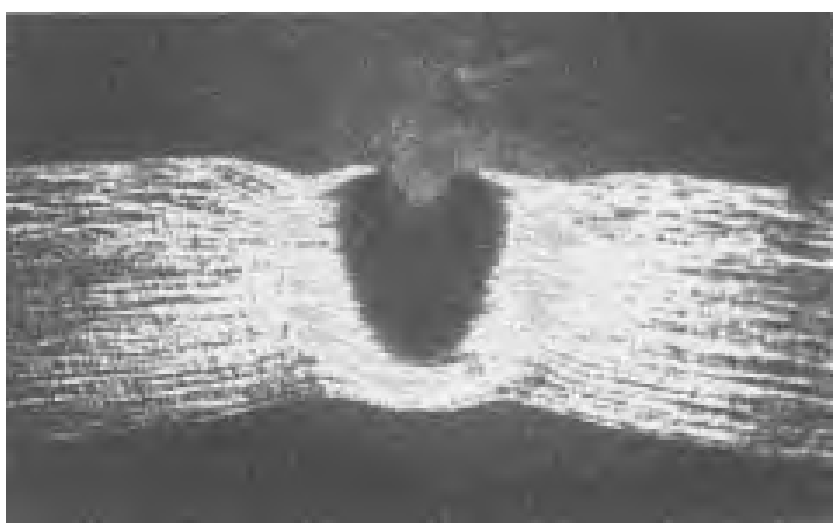

(b)

Fig. 2. (a) In vitro OCT image of a porcine cornea after being treated by a laser and (b) a Sirius-red-stained histology of the same specimen. The dimensions in the OCT image are lateral $4 \mathrm{~mm}$ and axial $1.7 \mathrm{~mm}$. The shape and the distribution of the laser-coagulation zone in both pictures correspond well except the overall shrinkage of cornea thickness, which is an artifact induced by histological preparation.

\section{A. In Situ Visualization of Biological Tissues with OCT}

Laser thermal keratoplasty (LTK) is a new technique for correction of refractive errors by the use of defined laserinduced peripheral corneal shrinkage which adjusts the central corneal curvature. Fig. 2(a) and (b) shows an in vitro crosssectional OCT image and the related light microscopy of a porcine cornea after laser coagulation. Although the light microscopy shows more microstructural details than the OCT image, the thermal-induced effects coincide fairly well in both pictures. On the other hand, OCT images are free of artifacts induced by histological processing and show the changes at the coagulation spot as well as the curvature of the cornea outside the coagulated sites. OCT seems to be a promising technique for noninvasive, in vivo evaluation of LTK effects which may provide more information than computer keratoscopy. Further laboratory study is in process.

Further improvement of the OCT system could lead to a method to detect invasion of the tumors into the lamina propria, submucosa, and muscularis layers.

Fig. 3(a) shows an in vivo OCT image of a porcine bladder with two laser-coagulated spots. Fig. 3(b) shows the corresponding $50-\mathrm{MHz}$ ultrasound image. During both measurements, the bladder was stretched and laser coagulation was

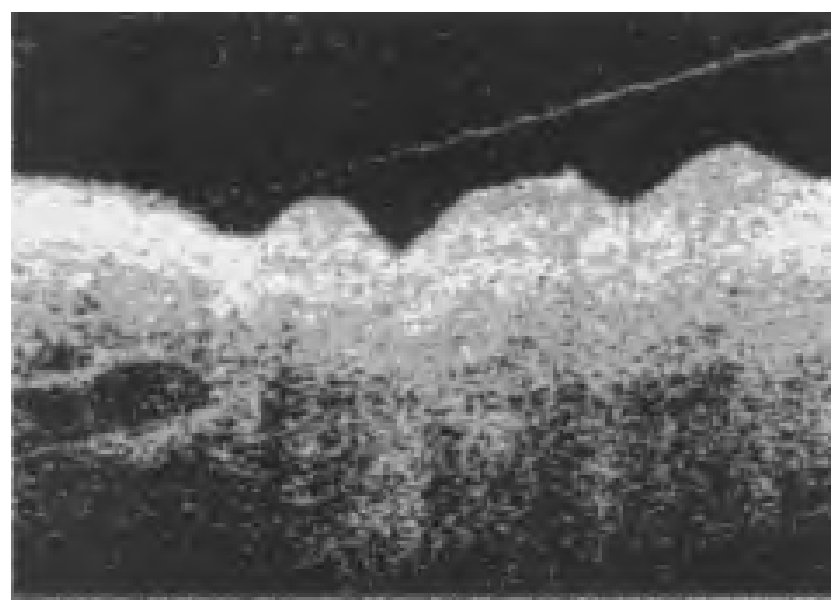

(a)

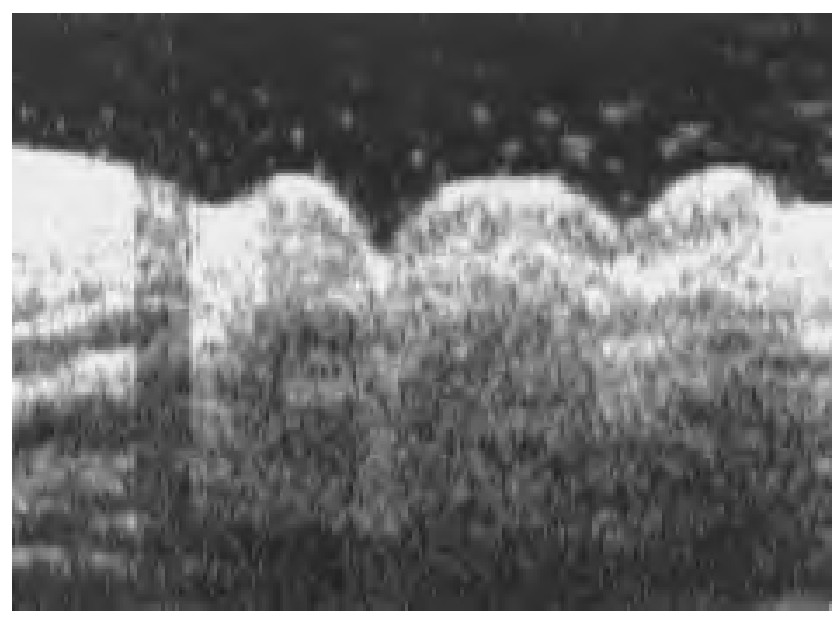

(b)

Fig. 3. (a) In vitro OCT image of a porcine bladder after laser coagulation and (b) $50-\mathrm{MHz}$ ultrasound image of the same specimen. Two coagulation spots were made to align both OCT and ultrasound lateral scans. The dimensions of both pictures are lateral $5 \mathrm{~mm}$ and axial $2 \mathrm{~mm}$. The overall coagulation area, and especially the dark structures in the OCT image on the left, coincide closely with those of the ultrasound in (b). The vertical band in (b) is an artifact because of an air bubble in the water above. The low-scattering epithelium is not shown because of detachment during sample preparation. The Lamina propria, about $400 \mu \mathrm{m}$ thick, is shown as a high signal region in OCT in (a), whereas the coagulation area shows a lower signal.

used to navigate the OCT scan. It can be seen that morphological details in mucosa and submucosa cannot be resolvable due to strong light scattering of bladder tissue. Structural differences such as the lamina propria $(\approx 400 \mu \mathrm{m}$ thick $)$ can be seen as a high signal in Fig. 3(a), and some structures, especially the dark areas, correlate well to those in the ultrasound image [Fig. 3(b)]. Unfortunately, the very fragile lowscattering epithelium on top of the bladder wall was destroyed during laser coagulation and the sample preparation. It is interesting to observe that the OCT signal in the coagulated area is actually lower than in the surrounding area even though the coagulated tissue appears to be brighter and more diffusive. This may be because laser coagulation causes tissue to become more homogeneous, which will lead to lower OCT modulation according to the model calculations (1). Further studies are required to analyze laser-induced scattering changes. 


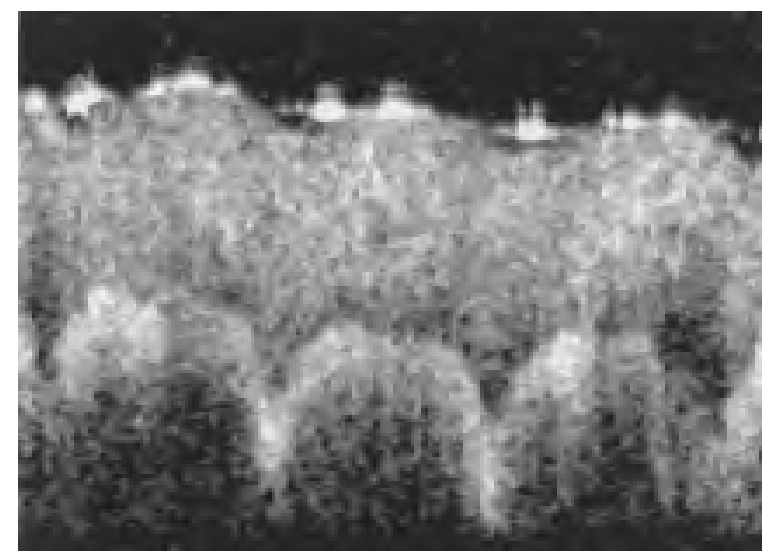

(a)

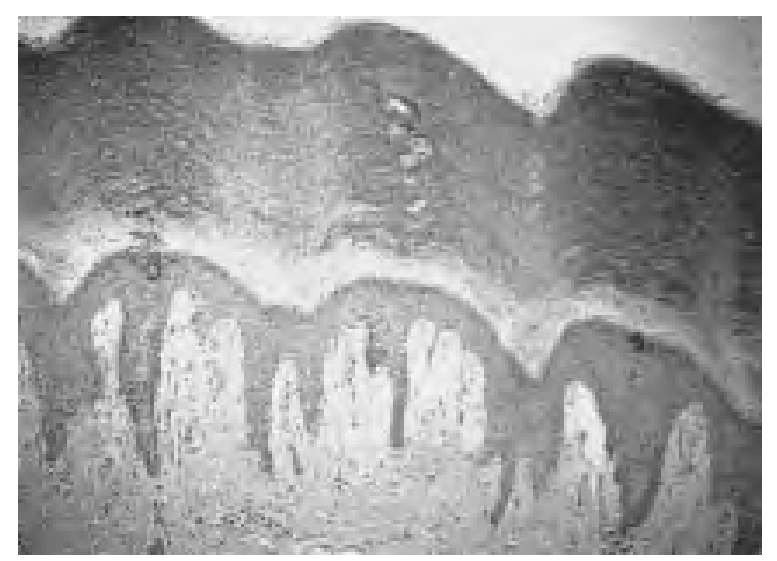

(b)

Fig. 4. (a) In vivo OCT image of a human fingertip and (b) a HE-stained histology. The dimensions of both pictures are lateral $2 \mathrm{~mm}$ and axial $1.3 \mathrm{~mm}$. The skin was treated with glycerin to enhance OCT contrast. The functional structures such as the $450-\mu \mathrm{m}$-thick stratum corneum followed by a thin layer (dark stripe) and the living epidermis (high signal) which corresponds well to the structures shown by the histology.

High-resolution visualization of living skin is highly demanded not only for clinical diagnostics of skin diseases but also for skin protection and cosmetics. Confocal microscopy has been successfully utilized to visualize cells of superficial skins, i.e., the epidermis. However, multiple scattering limits the penetration depth to $200-300 \mu \mathrm{m}$. On the other hand, imaging the structures at even slightly deeper layers, e.g., the conjunction of epidermis and dermis such as the stratum basale and the papillary layers ,are very interesting for studies on development of skin tumors. Fig. 4(a) shows an in vivo OCT image of a human fingertip. The first layer, which is about $450 \mu \mathrm{m}$, is the thick stratum corneum. The dark thin stripe below may be the translucent stratum lucidum, and the higher signal region below is the living epidermis. The corresponding light microscopy section is shown in Fig. 4(b). It can be seen that the structures in OCT image and light microscopy correlate well. During the measurement, the skin was treated with glycerin to enhance transparency.

Analyzing the above examples, we can summarize that because of multiple light scattering and a coherence length longer than most cell diameters, OCT can provide less microscopic details than light microscopy; however, it can show structures which correlate with ultrasound and light microscopy to a depth of about $600 \mu \mathrm{m}$ currently. Therefore, OCT may be used for imaging of living tissues like skin and bladder and for noninvasive diagnostics of superficial lesions.

\section{B. Preliminary Study for Skin Diagnostics}

Some preclinical measurements with patients and volunteers have been done to study the possible applications of OCT in dermatology. Fig. 5(b) shows a cross-sectional OCT image across a nevus cell nevus in the upper dermis of a female forearm. Afterwards, a $50-\mathrm{MHz}$ ultrasound image was performed and a biopsy was taken [Fig. 5(a) and (c)]. Good correlation can be found among these three pictures, especially between OCT and histology. Histology always shows more details about the nevus cells which are aggregated in nests in the upper dermis. However, it is interesting to see that OCT can detect the nevus which appears as a dark shadow in Fig. 5(a). In addition, OCT shows a high signal under the surface only at the nevus region. This is caused by the basal hyperpigmentation induced by melanin inside the epidermis. Similar effects have been found by Rajadhyaksha et al. [23] in confocal microscopy and also in our preliminary OCT results for carcinoma, which suggests that melanin shows higher scattering and better contrast. Further study on melanin-related phenomena will be done.

Fig. 6(a) and (b) shows OCT images of a virus wart, molluscum cantagiosum, before and three days after operation. A light microscopy image of a similar sample is shown in Fig. 6(c). This is virus induced. The OCT image shows fairly good correlation with light microscopy in that a hyperproliferative and acanthotic epidermis surrounds a horny granule. The OCT image shows hyperkeratosis as a dark area surrounded by a bright one induced by the epidermis. It can be seen in the OCT image of Fig. 6(b) that the wart is removed and that the epidermis becomes normal.

\section{CONCLUSION}

In optically translucent media, coherence-gated imaging, e.g., OCT, has longitudinal and lateral resolutions of about $10 \mu \mathrm{m}$ and a dynamic range of $100 \mathrm{~dB}$. In optically turbid tissues, this technique detects the pathlength-resolved backscattering, i.e., retroreflectance which is originated from the local reflecting sites with index-of-refraction discontinuity. Therefore, it is inherently sensitive to the microstructural difference. However, as most biological tissues are characterized by the single backscattered light, multiple scattered light can significantly influence the measured signal. The imaging contrast and the exceptional resolution of OCT are finally limited by the scattering-related noise rather than shot noise. This technique can be used to provide high-resolution imaging of either low-scattering or high-scattering superficial tissues which may include eye, skin, and some other tissues such as bladder, bronicle, and vascular systems which are accessible endoscopically.

This paper is aimed at studying the capabilities of OCT for high-resolution, in vivo imaging of living tissues and noninvasive tumor diagnostics. From the OCT images presented in this 


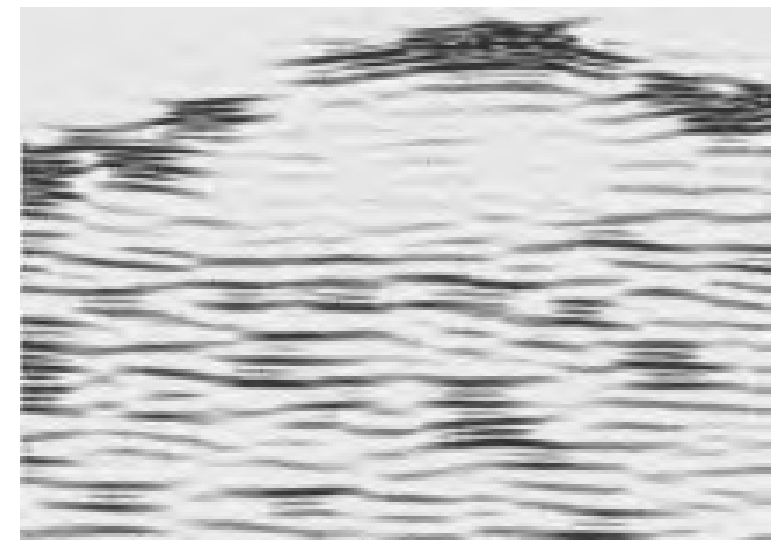

(a)

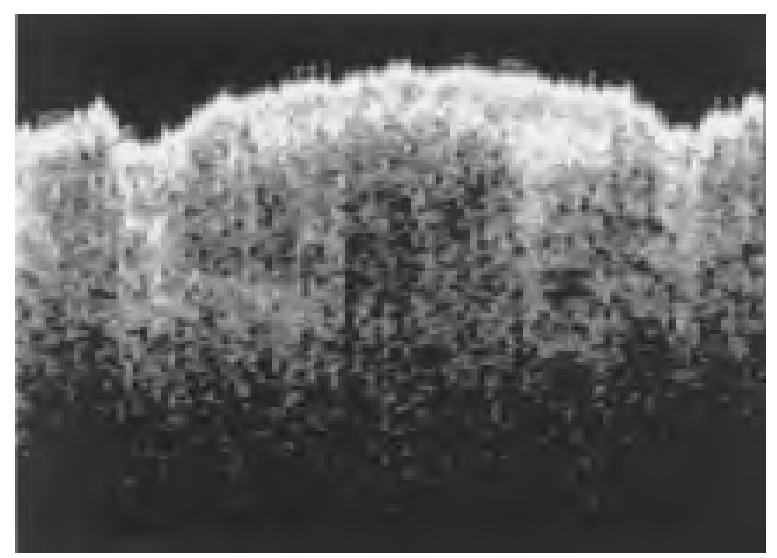

(b)

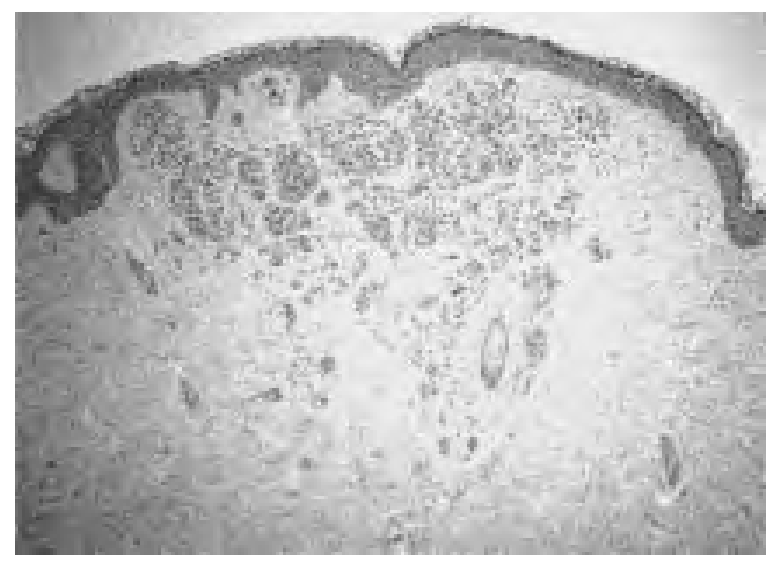

(c)

Fig. 5. (a) 50-MHz ultrasound, (b) in vivo OCT image of a nevus, and (c) HE-stained histology of the same specimen. The dimensions of the three pictures are lateral $2 \mathrm{~mm}$ and axial $1.3 \mathrm{~mm}$. The nevus cells are aggregated in nests in the upper dermis of a female forearm. The overall nevus distribution shown as a dark shadow or "echo poor" in the ultrasound in (a) and the OCT image in (b) correspond to the nevus-cell region in the histology in (c). Note that there is a second thin high signal layer under the surface of the nevus in the OCT image. This is caused by a high melanin concentration at the end of the epidermis.

paper, we can conclude that OCT provides less microstructural details such as the cellular and even subcellular structures than light microscopy. This is caused by the speckle appearance due to the path randomization by scattering and a coherence length $L_{c}$ larger than most cell sizes. OCT can be used for

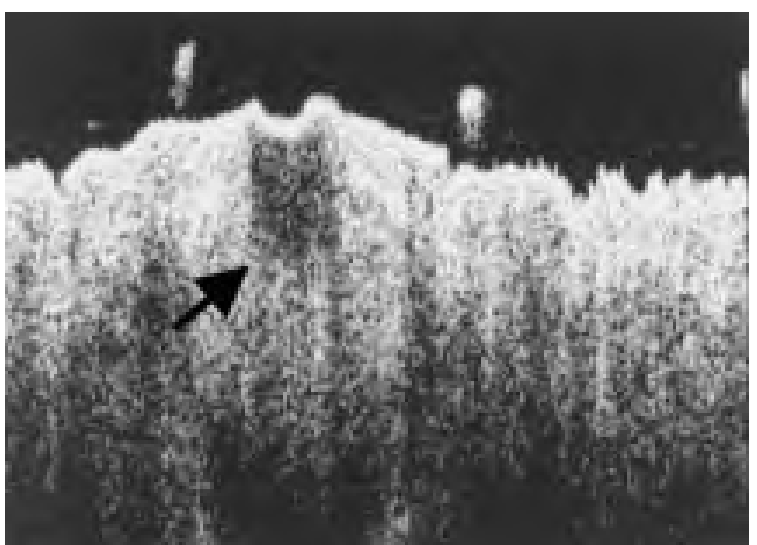

(a)

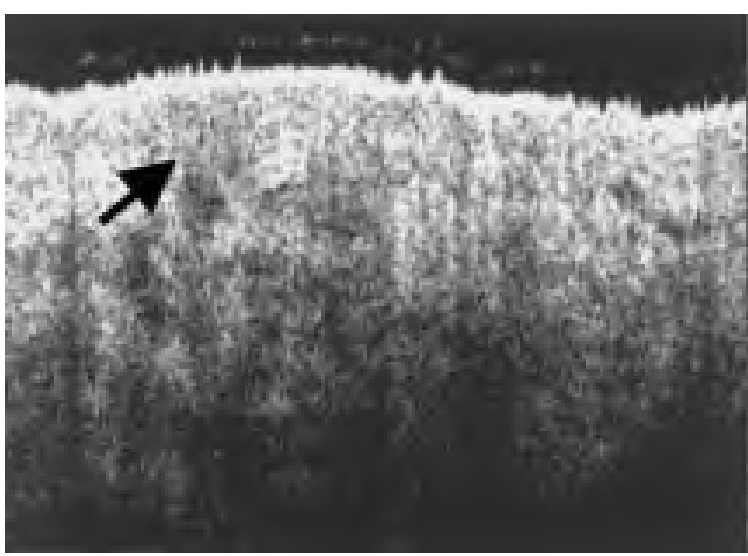

(b)

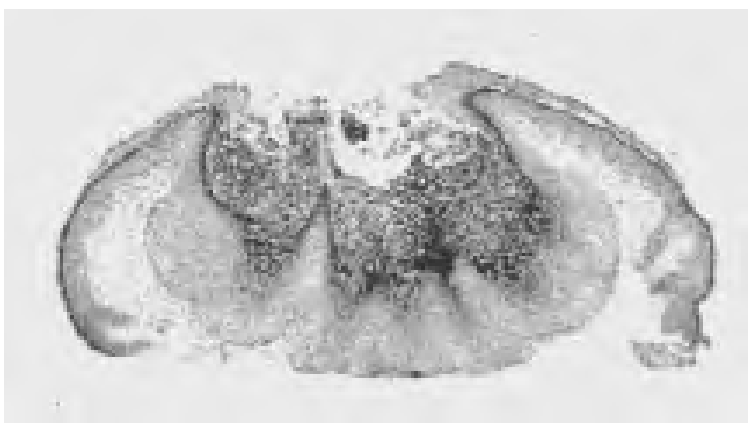

(c)

Fig. 6. In vivo image of a wart, moluscum contagiosum, at the forearm of a five-year-old girl (a) before and (b) three days after operation. (c) A HE-stained histology of a similar specimen. The dimensions of both OCT images are lateral $4 \mathrm{~mm}$ and axial $1.03 \mathrm{~mm}$. OCT illustrates clearly that the horny granule which is a dark area in (a) above the arrow is surrounded by a hyperproliferative and acanthotic epidermis. After operation, the granule is removed and the epidermis becomes normal (arrow) in (b).

functional in vivo imaging. For example, the lamina propria and mucosa of bladder and the epidermis and dermis of skin scanned by OCT are found to be inconsistent with the corresponding light microscopy and $50-\mathrm{MHz}$ ultrasound. The capability of this technique for tissue malformation detection has been shown. An interesting effect, the melanin-induced high backscattering area has been clearly shown in an OCT image. These results of our preliminary clinical study show the potential applications of OCT as an optical biopsy for noninvasive tumor diagnostics. Further study should be done 
to provide a profound understanding of contrast differences between normal and cancerous tissues. The speed of the imaging system should be increased to minimize the influence of tissue movement. The contrast and penetration depth of OCT can be further improved by use of light sources at longer wavelengths.

\section{ACKNOWLEDGMENT}

The authors thank M. Vogel from the University of Lübeck, who contributed the histology shown in Fig. 2(b). The authors also thank Taberna pro Medicum, D-21335 Lüneburg, Germany, for the 50-MHz ultrasound measurement shown in Fig. 5(a).

\section{REFERENCES}

[1] E. A. Hoffman, Ed., "Advances in optical imaging and photon migration," Proc. Soc. Photo-Opt. Instrum. Eng., Tech. Dig., vol. 2433, pp. 27-28, 1995.

[2] B. Chance, K. Kang, and E. Sevick, "Photon diffusion in breast and brain: Spectroscopy and imaging," Opt. Photon. News, vol. 4, no. 10 pp. 8-13, 1993.

[3] D. A. Benaron and D. K. Stevenson, "Optical time-of-flight absorbance imaging in biomedical media," Science, vol. 259, pp. 1443-1446, 1993.

[4] F. Liu, K. M. Yoo, and R. R. Alfano, "Ultrafast laser-pulse transmission and imaging through biological tissues," Appl. Opt., vol. 32, pp. 554-558, 1993.

[5] R. Berg, O. Jarlman, and S. Svanberg, "Medical transillumination imaging using short-pulse diode lasers," Appl. Opt., vol. 32, pp. 572-579, 1993.

[6] J. Weng, M. Z. Zhang, K. Simon, and B. Chance, "Measurement of biological tissue metabolism using phase modulation spectroscopic technology," in Time-Resolved Spectroscopy and Imaging of Tissues, B. Chance and A. Katzir, Eds., in Proc. Soc. Photo-Opt. Instrum. Eng., 1991, vol. 1431, pp. 161-170.

[7] L.-H. Wang, S. L. Jacque, and X.-M. Zhao, "Continuous-wave ultrasonic modulation of scattered laser light to image objects in turbid media," Opt. Lett., vol. 20, pp. 629-631, 1995.

[8] J. R. Lakowicz and K. W. Berndt, "Lifetime-selective fluorescence imaging using an RF phase-sensitive camera," Rev. Sci. Instrum., vol. 62 , no. 7 , pp. $1727-1734,1991$.

[9] J. R. Lakowicz, K. Nowaczyk, K. W. Berndt, and M. Johnson, "Fluorescence lifetime imaging," Anal. Biochem., vol. 202, pp. 316-330, 1992.

[10] J. M. Schmitt, A. Knuettel, and M. Yadlosky, "Confocal microscopy in turbid media," J. Opt. Soc. Amer. A, vol. 11, pp. 2226-2235, 1994.

[11] D. Hang, E. A. Swanson, C. P. Lin, J. S. Schuman, W. G. Stinson, W. Chang, M. R. Hee, T. Flotte, K. Gregory, C. A. Puliafito, and J. G. Fujimoto, "Optical coherence tomography," Science, vol. 254, pp. 1178-1181, 1991

[12] Y. Pan, S. Arlt, R. Birngruber, and R. Engelhardt, "Optical coherence tomography in turbid tissue: theoretical analysis and experimental results," Optical and Imaging Techniques for Biomonitoring, H.-J. Foth and R. Marchesini, Eds., in Proc. Soc. Photo-Opt. Instrum. Eng., 1995, vol. 2628 , pp. 239-268.

[13] J. A. Izatt, M. R. Hee, G. M. Owen, E. A. Swanson, and J. G. Fujimoto, "Optical coherence microscopy in scattering media," Opt. Lett., vol. 19, pp. 590-592, 1994.
[14] C. K. Hitzenberger, W. Drexler, and A. F. Fercher, "Measurement of corneal thickness by laser Doppler interferometer," Invest. Ophthalmol. Vis. Sci., vol. 33, pp. 98-103, 1992.

[15] B. Bouma, G. Tearney, S. Boppart, M. R. Hee, M. Brezinski, and J.G. Fujimoto, "High-resolution optical coherence tomographic imaging using a mode-locked Ti: $\mathrm{Al}_{2} \mathrm{O}_{3}$ laser source," Opt. Lett., vol. 20, pp. 1486-1488, 1995.

[16] Y.-T. Pan, R. Birngruber, J. Rosperich, and R. Engelhardt, "Lowcoherence optical tomography in turbid tissue: Theoretical analysis," Appl. Opt., vol. 34, pp. 6564-6574, 1995.

[17] M. J. Yadlowsky, J. M. Schmitt, and R. F. Bonner, "Multiple scattering in optical coherence microscopy," Appl. Opt., vol. 34, pp. 5699-5707, 1995.

[18] Y. Pan, R. Birngruber, and R. Engelhardt, "Contrast limits of coherencegated imaging in scattering media," Appl. Opt., to be published.

[19] J. Schmitt, M. Yadlowsky, and R. Bonner, "Subsurface imaging of living skin with optical coherence microscopy," Dermatology, vol. 191, pp. 93-98, 1995.

[20] G. Tearney, S. Boppart, B. Bouma, M. E. Brezinski, N. J. Weissmann, J. F. Southern, J. G. Fujimoto, "Scanning single-mode fiber optic catheterendoscope for optical coherence tomography," Opt. Lett., vol. 21, pp. 543-545, 1996.

[21] J. M. Schmitt, A. Knüttel, and M. A. Eckhaus, "Optical coherence tomography of a dense tissue: Statistics of attenuation and backscattering," Phys. Med. Biol., vol. 39, pp. 1705-1720, 1994.

[22] J. G. Fujimoto, M. E. Brezinski, G. J. Tearney, A. S. Boppart, B. Bouma, M. R. Hee, J. F. Southern, E. A. Swanson, "Biomedical imaging and optical biopsy using optical coherence tomography," Nature Medicine, vol. 1, pp. 970-972, 1995.

[23] M. Rajadhyaksha, M. Grossmann, D. Esterowitz, R. H. Webb, and R R. Anderson, "In vivo confocal scanning microscopy of human skin: Melanin provides strong contrast," J. Investigative Dermatol., vol. 104, pp. 946-952, June 1995.

Y. Pan, photograph and biography not available at the time of publication.

E. Lankenau, photograph and biography not available at the time of publication.

J. Welzel, photograph and biography not available at the time of publication.

R. Birngruber, photograph and biography not available at the time of publication.

R. Engelhardt, photograph and biography not available at the time of publication. 